\title{
Comparison of Outcomes Based on Graft Type and Tunnel Configuration for Primary Ulnar Collateral Ligament Reconstruction in Professional Baseball Pitchers
}

Timothy B. Griffith, MD², Christopher S. Ahmad, MD², Michael G. Ciccotti, MD³, John D'Angelo4 , Joshua S. Dines, MD5, David W. Altchek, MD ${ }^{6}$, Christopher L. Camp, MD

${ }^{1}$ Peachtree Orthopedics, Atlanta, GA, USA, ${ }^{2}$ Columbia University, New York, NY, USA, ${ }^{3}$ Rothman Institute at Thomas Jefferson University Hospital, Philadelphia, PA, USA, ${ }^{4}$ Major League Baseball, New York, NY, USA, ${ }^{5}$ Hospital for Special Surgery, uniondale, NY, USA, ${ }^{6}$ Hospital for Special Surgery, New York, NY, USA, ${ }^{7}$ Mayo Clinic, Rochester, MN, USA.

Objectives: Professional baseball pitchers are at high risk for tears of the ulnar collateral ligament (UCL) of the elbow, often requiring subsequent surgical reconstruction. Despite acceptable published return to play outcomes, multiple techniques and graft types have been described. There is a paucity of clinical data in the current literature comparing UCL reconstruction surgical technique and graft type. Even less is known about the risks for subsequent injury, surgery, or revision UCL reconstruction. Accordingly, this study compares UCL reconstruction outcomes based on tunnel configuration and graft type.

Methods: Following approval from our institutional review board and Major League Baseball (MLB), 566 professional baseball pitchers who underwent UCL reconstruction between 2010 and 2014 were identified and included. The following patient demographics were analyzed: age, pitching role (starter vs. reliever), level of play (MLB vs. Minor League Baseball [MiLB]), and throwing side dominance. Surgical factors analyzed included reconstruction technique (Docking vs. Modified Jobe), graft type (palmaris longus autograft vs. gracilis autograft), and concomitant procedures. Primary outcome measures consisted of: the ability to return to play at any level (RTP), to return to the same level of play (RSL), the time to return, subsequent elbow injuries, and the need for subsequent or revision elbow surgery. The impact of the patient and surgical factors on outcomes were analyzed using multivariate linear and logistic regression modeling.

Results: The overall RTP was $79.9 \%$ and RSL was $71.2 \%$. There were no significant differences in the time to RTP or RSL based on reconstruction technique or graft type. RTP rates were similar for the Docking vs. Modified Jobe techniques $(80.1 \%$ vs. $82.4 \%$; $p=0.537)$ and for the two primary graft types $(83.1 \%$ for palmaris vs. $80.7 \%$ for gracilis; $p=0.596)$. The risk of subsequent elbow surgery was $10.5 \%$ for the Docking Technique vs. $14.8 \%$ for the Modified Jobe $(p=0.203)$; and the risk for subsequent UCL revision reconstruction surgery was $2.9 \%$ vs. $6.2 \%$ for the Docking vs. Modified Jobe Techniques, respectively $(p=0.128)$. Significant trends towards an increasing use of palmaris autograft $(p=0.023)$ and the docking technique $(p=0.006)$ were observed. MLB pitchers were more likely than MiLB pitchers to RTP $(p<0.001)$ and to RSL $(p<0.001)$, but they required a longer time to return (mean difference 35 days; $p=0.039$ ), had a higher likelihood of subsequent elbow (OR 3.58; $95 \% \mathrm{Cl} 2.055$ to 6.231 ; $p<0.001$ ) and forearm injuries (OR 5.695; $95 \% \mathrm{Cl} 1.99$ to $16.302 ; p=0.004$ ), but not subsequent elbow surgery. No specific variables were noted to be predictive of subsequent elbow or revision surgery in the multivariate analysis. Conclusion: Surgical outcomes in professional baseball players are not significantly influenced by ulnar collateral ligament reconstruction technique or graft type usage. Major League players are more likely to RTP and RSL, but they have a higher frequency of subsequent elbow and forearm injuries. Both the Docking Technique and palmaris autograft are increasing in popularity amongst surgeons treating professional baseball players.

The Orthopaedic Journal of Sports Medicine, 6(7)(suppl 4)

DOI: $10.1177 / 2325967118 S 00087$

CThe Author(s) 2018

This open-access article is published and distributed under the Creative Commons Attribution - NonCommercial - No Derivatives License (http://creativecommons.org/licenses/by-nc-nd/4.0/), which permits the noncommercial use, distribution, and reproduction of the article in any medium, provided the original author and source are credited. You may not alter, transform, or build upon this article without the permission of the Author(s). For article reuse guidelines, please visit SAGE's website at http://www.sagepub.com/journals-permissions. 